\title{
POLARIZATION OBSERVATIONS OF SELECTED RADIO GALAXIES AT 327 MHZ
}

\author{
ANTHONY G. WILLIS \\ Athabasca University, Box 10000, Athabasca, Alberta, Canada \\ CHRISTOPHER P. O'DEA \\ Netherlands Foundation for Research in Astronomy \\ PO Box 2, 7990 AA Dwingeloo, The Netherlands
}

\begin{abstract}
We have measured the distribution of polarized emission from the radio galaxies NGC315, NGC6251, DA240, 3C236 and 3C326 at $327 \mathrm{MHz}$ (92 cm wavelength) with the Westerbork Synthesis Radio Telescope (WSRT). These radio galaxies were all selected for observation because they exhibited degrees of polarization as high as 60 or 70 percent at $610 \mathrm{MHz}$ (49 $\mathrm{cm}$ wavelength) and because they all have angular sizes greater than about 20 arcmin. Therefore they are still well-resolved by the WSRT at $92 \mathrm{~cm}$ wavelength. All sources in the sample exhibit significant linear polarization at $92 \mathrm{~cm}$ wavelength. Some depolarization does occur in NGC315 and 3C326 but parts of 3C236, DA240 and NGC6251 appear not to depolarize to $92 \mathrm{~cm}$. Assuming an ordered, equipartition magnetic field, we find that the density of thermal depolarizing material in these radio galaxies is very low, typically about $1 \times 10^{-5} \mathrm{~cm}^{-3}$ or less. Therefore these radio galaxies may represent distributions of pure magnetic fields and relativistic particles on scales of several hundred kiloparsecs or more.
\end{abstract}

\section{Observations and Data Reduction}

All galaxies were observed by the WSRT in full redundancy mode; the correlator sampled both parallel and crossed dipole configurations. At $327 \mathrm{MHz}$ electric vector position angles can be rotated from their intrinsic position angles by many tens of degrees (more than 100 degrees is not uncommon) because of Faraday rotation within the ionosphere. Therefore all observations were first corrected for ionospheric Faraday rotation using the method developed at Dwingeloo by Spoelstra (1981).

\section{Results}

NGC315: At $92 \mathrm{~cm}$ the polarization along the jet of NGC315 remains roughly constant at about 15 to 20 percent. This contrasts with an average degree of polarization of about 35 to 40 percent at $21 \mathrm{~cm}$ (Willis et al., 1981), and which increases with increasing distance 
from the nucleus. We suggest that the jet is entraining thermal matter as the distance from the nucleus increases and that the density of this thermal matter is about is about $2 \times 10^{-4} \mathrm{~cm}^{-3}$.

3C236: Both lobes of this giant radio galaxy have significant $92 \mathrm{~cm}$ polarization. The leading edge of the southeast lobe has a degree of polarization of about 11 percent. This value is consistent with that found in previous $21 \mathrm{~cm}$ WSRT polarization measurements (Strom and Willis, 1980). The lack of depolarization to $92 \mathrm{~cm}$ implies a thermal density of $<1 \times 10^{-5} \mathrm{~cm}^{-3}$. Some depolarization does occur in the interior parts of the radio lobes of $3 \mathrm{C} 236$. The thermal matter density would have to be about $2.5 \times 10^{-5} \mathrm{~cm}^{-3}$ to cause the observed depolarization.

3C326: The amounts of polarized emission found (up to 10 percent at the western end of the source), and the corresponding density of thermal depolarizing material, about $3 \times 10^{-5}$ $\mathrm{cm}^{-3}$ are consistent with that expected from the earlier WSRT study of Willis and Strom (1978).

NGC6251: The northwest lobe exhibits degrees of polarization as high as 70 percent and polarized radiation is detected from most parts of the lobe. In contrast, the southeast lobe does not seem to have much polarized radiation. A comparison of the distribution of polarization at $92 \mathrm{~cm}$ with WSRT $49 \mathrm{~cm}$ results (Willis, Wilson and Strom, 1978) indicates that there is probably no depolarization to $92 \mathrm{~cm}$ in the northwest lobe. Therefore the density of any thermal material must be very low, well under $1 \times 10^{-5} \mathrm{~cm}^{-3}$.

DA240: DA240 shows considerable polarization at $92 \mathrm{~cm}$. The outer lobes have typical degrees of polarization of about 20 percent. This is down from the values of up to 60 percent that are seen at shorter wavelengths. Of particular interest is the bright hot spot in the eastern lobe of DA240. This feature seems to maintain a constant degree of polarization of about 20 percent over the wavelength range $6 \mathrm{~cm}$ (Tsien, 1982) to $92 \mathrm{~cm}$ and, again, the density of any thermal material in this hot spot must be very low, $<1 \times 10^{-5} \mathrm{~cm}^{-3}$.

\section{Acknowledgements}

AGW thanks NSERC for operating and equipment grants. The Westerbork Telescope is operated by the Netherlands Foundation for Research in Astronomy, which is financially supported by the Netherlands Organisation for Scientific Research (NWO).

\section{References}

Spoelstra, T.A.Th. (1981) NFRA Internal Technical Report 162.

Strom, R.G. and Willis, A.G. (1980) Astron. Astrophys. 85, 36.

Tsien, S.C. (1982) Monthly Notices Roy. Astron. Soc. 200, 377.

Willis, A.G. and Strom, R.G. (1978) Astron. Astrophys. 62, 375.

Willis, A.G., Strom, R.G., Bridle, A.H., Fomalont, E.B. (1981) Astron. Astrophys. 95250. 